\title{
Using Online Carbon Calculators for Participation in Local Climate Initiatives
}

\author{
Georg Aichholzer, Doris Allhutter, and Stefan Strauß \\ Austrian Academy of Sciences, Institute of Technology Assessment (ITA), Strohgasse 45, \\ 1030 Vienna, Austria \\ \{aich, dallhutt, sstrauss\} @oeaw.ac.at
}

\begin{abstract}
The paper reviews various eco-feedback systems including carbon calculators and discusses how different disciplinary approaches conceptualise and explain anticipated impacts of these systems. The European collaborative research project e2democracy investigates how citizen participation combined with long-term $\mathrm{CO}_{2}$ monitoring and feedback can contribute to achieve local climate targets. Empirical results from local climate initiatives in Austria, Germany and Spain show positive effects in terms of learning about $\mathrm{CO}_{2}$ impacts, increased awareness, enhanced efforts and guidance as well as individual empowerment leading to slightly reduced $\mathrm{CO}_{2}$ emissions. The findings highlight that a combined approach integrating eco-feedback and (e-)participation is promising to foster sustainability.
\end{abstract}

Keywords: citizen participation, climate protection, carbon calculator, ecofeedback, environmental impacts.

\section{Introduction}

Citizen participation has become an important issue of sustainability policy as the concept "environmental democracy" indicates [1]. Access to environmental information and the integration of all stakeholders in decision-making processes are acknowledged principles of environmental democracy. The urgency of actions further suggests public participation as a strategic resource and practice in fighting climate change. Accordingly, various forms of citizen engagement in climate issues are spreading [2, 3, 4]. However, citizen participation in climate policies, particularly via electronic means, is still largely unexplored. Viewed from a functional perspective such a participation approach holds potential, which ranges from information sharing, awareness raising and mobilisation of collective effort to collaborating on policy decisions, their implementation, and thus, enhanced problem solving.

Recent developments in the field of ecological-feedback technologies offer potential synergies with participatory approaches. Eco-feedback provides various sorts of information, which enhance the understanding of one's patterns and impacts of energy consumption, with the intended effect to stimulate a rethinking of habits and change to pro-environmental behaviour [5]. Carbon calculators are of special 
relevance for climate protection strategies as they inform about the "carbon footprint" of individuals, households or businesses. Existing reviews acknowledge the value of such devices but suggest improvements with regard to accuracy, transparency, meaningful guidance and feedback and opportunities for connecting with other users $[6,7]$. The Internet offers advantages to make these tools effective information and learning systems, in particular in combination with a citizen participation approach.

This paper aims at exploring the potential of eco-feedback in participatory approaches of local governments. The European collaborative research project "e2democracy" (environmental electronic democracy; http://www.e2democracy.eu) provides empirical results from a survey among citizens who participated in climate initiatives in six cities. In this paper our research questions are: Do citizens use the information offered? How do they assess the value of eco-feedback? To what extent does the participatory setting support individual motivations and collective efforts? What are the impacts regarding local climate targets? The subsequent sections introduce eco-feedback systems (2); describe the empirical setting of citizen participation and the role of the online carbon calculator (3); sketch the research methods (4); present the findings (5) and summarise the conclusions (6).

\section{Electronic Eco-Feedback Systems}

Eco-feedback systems collect data on individual or group behaviours from a variety of domains including energy consumption, water usage, transportation, garbage and recycling behaviours and product purchases. Electronic forms range from energy monitoring and ambient energy awareness technologies ${ }^{1}$ to theme-specific edutainment applications and social websites. ${ }^{2}$ New tools include sensing systems for activities such as power consumption or waste behaviour which feedback data e.g., via mobile phones $^{3}$ and smart meters that provide (near) real-time data on electricity, gas and water usage. Darby [11] discerns various forms and contents of feedback, including raw data such a as meter-data or data from cost plugs ('direct feedback'), processed data such as bills that integrate comparative information or detailed energy reports ('indirect feedback'), and information integrating different data sources by using software such as carbon calculators ('energy audits'). Froehlich et al. [12] distinguish between 'low-level feedback' providing details about how to improve specific consumption behaviours, and 'high-level feedback', which is summative and supports the improvement of one's performance towards a specific goal or in comparison to other users. At large, eco-feedback intends to offer a sort of learning tool which enables users to reflect on their energy consumption behaviours, underlying valuesystems and environmental impacts through feedback and experimentation.

\footnotetext{
${ }^{1}$ E.g., 'WaterBot', a water tap which provides information feedback on water usage [8].

${ }^{2}$ E.g, 'BinCam' monitors food waste and recycling behaviour [9].

${ }^{3}$ E.g., 'UbiGreen' senses and feeds back data to encourage green transportation habits [10].
} 


\subsection{Theoretical Background}

Integral aims of eco-feedback are enhanced control over energy usage, consumptionrelated awareness [11,13] and support in making energy practices accountable. Darby [11] suggests a combination of different feedback approaches, in particular historic feedback on previous consumption periods, may contribute to achieving learning effects and creating "tacit knowledge".

Research in human-computer interaction and ubiquitous computing links such approaches to the question how a technology should present relevant information and how it should interact with people to encourage sustainable lifestyles [14, 15]. A crucial point is whether and how such systems succeed in transforming individual and shared responsibility for environmental issues into the ability to implement appropriate measures and to change related behaviours (despite the evidence of a 'value-action gap') [16]. Focussing on the ability to act on the basis of better information presupposes that knowledge and related values do translate into action. However, relevant capacities are tied to personal and household behaviours as well as to structural and material conditions in which individual and community practices are embedded and with which they co-emerge [17].

According to Froehlich et al. [12] eco-feedback technology is based on the assumption that raising awareness and knowledge about the way everyday routines affect the environment may bridge the "environmental literacy gap" and lead to behavioural changes. Based on a survey of 89 papers from environmental psychology the authors identify two basic models of pro-environmental behaviour: rational choice models, which consider environmental behaviour mainly as driven by selfinterest, and norm-activation models, which take social motives as central basis for action. Under rational choice models, the authors subsume various models which suggest that (1) favourable attitudes translate into respective behaviours, that (2) issue knowledge and information on appropriate actions are crucial for attitudes to actually trigger favourable behaviours, and finally, that (3) consumers adopt sustainable behaviours that are economically advantageous and discount factors such as convenience, habit and social norms. In contrast, norm-activation models highlight the importance of moral or personal norms and suggest that recognising the way in which one's environmental behaviours affect community goods and future generations, can activate moral obligations which may outplay individual perceptions of utility.

\subsection{Impacts of Eco-Feedback and Their Limits}

Recent studies in the smart metering context [e.g. 18, 19, 20] report energy savings in the ranges of $1.5-15 \%$ due to feedback technologies. The large span indicates limited comparability of findings though [18]. In general, higher figures result from smallscale studies with extremely motivated participants; also, evidence on the duration of savings is mixed but, in general, mostly positive.

Thaler/Sunstein [21] propose a behavioural economics perspective that directly addresses the relation between behaviour change and sustainable practices: it suggests offering a suitable "choice architecture" through appropriate information feedback 
that is to give "gentle nudges" in the direction of desired behaviours. The authors describe those decisions as most difficult, which have uncertain or delayed effects, provide little feedback or are ambiguously related with practical experience; a situation typically encountered in the context of energy consumption. Offering households information on their individual energy consumption, compared to previous periods and to the average consumption of their neighbourhood, together with positive and negative emoticons, showed positive effects on behaviour: households consuming above average decreased their consumption level, but belowaverage consumers increased their energy use significantly. Aside from this unintended "boomerang effect" the feedback of information and the opportunity for making comparisons seem to have served as a positive nudge at large.

However, there are also serious objections against the focus on pro-environmental behaviour change through information feedback and its viability. Criticising "the dominant paradigm of 'ABC' - attitude, behaviour, and choice", Shove [22] identifies blind spots of models which focus on the concept of choice and individual behaviour. This framing marginalises other possible approaches grounded in social theories of practice and transition. Theories of transition suggest calling into question the status quo: ... "relevant societal innovation is that [...] in which more sustainable regimes of technologies, routines, forms of know how, conventions, markets, and expectations take hold across all domains of daily life" [22, p. 1278]. In contrast to rational choice models and norm-activation models, theories of practices focus on the enactment of practices in specific social and temporal contexts and the emergent outcomes of such practices. In this spirit, approaches for assessing environmental practices of individuals need to consider how these practices co-emerge with socio-ecological systems and collective practices.

Stressing the complexity of behavioural issues in a similar way, ecological economists disapprove of the rational-choice approaches that widely dominate the literature in their community. Garmendia and Stagl, for example, highlight social learning as a key element of the route to sustainability - "a process that is going beyond the acquisition of mere factual knowledge" and that entails a "need to look beyond individual actors" [23, p. 1714]. According to the authors, relevant social learning processes include deliberation and should overcome individual and predefined interests and values to "create opportunities for a shared understanding and joint action" [23, p. 1713]. This perspective suggests that eco-feedback systems would gain from incorporating a mode of consumer or citizen participation that encourages the development of collective effort, of solidarity with a group and, in consequence, the adoption of collective interests as one's own.

\section{Case Study: Citizen Participation in Local Climate Policies}

The research project "e2democracy" has been studying a set of similar forms of citizen participation in climate policies at local government level in Austria, Germany and Spain. These climate initiatives allow us to analyse potentials and limits of ecofeedback in the context of a participatory design. 


\subsection{Common Organisational Characteristics}

All seven initiatives comprised similarly organised participation processes in different cities and regions: Bregenz and Mariazellerland (Austria); Bremen, Bremerhaven and Wennigsen (Germany); Zaragoza and Pamplona (Spain). The sites include small and large cities as well as rural communities, but share some common core elements: at each site local government, local companies and citizens agreed on the target to reduce $\mathrm{CO}_{2}$ emission levels by at least $2 \%$ per year; the participation process was carried out by a citizen panel working with local government on achieving or exceeding the agreed target; participation was projected to last up to two years; a common carbon calculator was used for individual $\mathrm{CO}_{2}$ balancing as a key tool; free choice of the mode of participation was practised - via traditional means (in person, via mail, telephone etc.) or via e-participation. Moreover, large-scale information measures via local media and kick-off events took place to spread invitations to all citizens plus project-related telephone surveys among the population to raise the awareness of the opportunity to participate.

Three types of interaction constituted the participation process: (1) provision of and access to information offering guidance on climate-friendly behaviour (regular newsletters, information via project website or on paper); (2) documentation of individual consumption data (via an online carbon calculator with instant production of individual $\mathrm{CO}_{2}$ balances, or via a personal " $\mathrm{CO}_{2}$ household book" on paper with subsequent calculation and transmission of $\mathrm{CO}_{2}$ balances by support staff); (3) various forms of theme-oriented meetings and exchange (e.g. group meetings with expert talks, group excursions, chats with experts, discussion platforms).

Providing participants with the possibility to individually monitor their energy consumption, get feedback and additional information, as well as exchange activities over a longer time period was meant to stimulate informed choices and to support responsible behaviour leading to reduced $\mathrm{CO}_{2}$ emissions. Depending on local agendas the participation processes started at different points in time. ${ }^{4}$ By April 2012 they lasted for two full years in Bremen, and for nearly two years in Austria and Spain.

\subsection{Common Features of Eco-Feedback via Carbon Calculator}

Most of the existing carbon tools break down the calculation by activity but estimate emission impacts only on an annual basis. The local climate initiatives studied by the e2democracy project employed an advanced carbon calculator adapted for regular bimonthly measurements over longer time periods. The calculator was an online tool for gathering, processing, storing and managing individual energy consumption data, and provided additional functionalities. Participants accessed the tool via the e2democracy portal, which allowed them to enter, process, calculate and continuously handle their individual $\mathrm{CO}_{2}$-balances. Additional functions included access to different kinds of information about regional organisation units, newsletters, hints for energy saving etc.

4 Bremen started the initiative in January 2010; Austrian and Spanish cities followed between April and autumn 2010, and the remaining two German cities began even later (Wennigsen, not yet included in the results, started last, in May 2011). 
Via a web forum, users were able to gain support for using the tool and discuss experiences with other users.

The calculator was based on four main activities (energy supply - including electricity and heating, mobility, nutrition, consumption). Corresponding data were entered bi-monthly. Help texts provided calculation examples and guided users through the application. As a starting point for managing and maintaining their balance, users entered their consumption data and behavioural patterns based on the previous year to create the baseline measurement. This was the reference point for the first entry of actual data two months later. In the subsequent bi-monthly measurements users were able to carry over data from previous rounds to speed up data entry and reduce overall effort. Individual feedback allowed users to reflect on their own behaviours and inherent energy consumption routines. Opportunities for self-reflection were enhanced by particular forms of presenting feedback information to allow participants to alter their behavioural patterns and develop more climatefriendly ways of life. The tool provided four major features:

a) Individual feedback: for each period entered, users got instant feedback on their corresponding $\mathrm{CO}_{2}$-balance (visualised by an interactive chart) broken down by activity and compared to the national average (see Figure 1);

b) Comparative feedback over time: changes of the $\mathrm{CO}_{2}$-balance (in total and per item) were visualised in different charts and tables (see Figure 2);

c) Comparative feedback with other groups: the line-chart showed the individual curves compared to regional panels and similar households; bar-chart and table showed individual values plus emoticons by activity over time.

d) Supporting information and learning opportunities: information on options for energy-saving; options for interactive experimentation with individual reduction targets; feedback on how this affected the balance (e.g. calculating the amount of $\mathrm{CO}_{2}$-reduction when saving $200 \mathrm{~km}$ per car or similar).

\begin{tabular}{|c|c|c|c|}
\hline \multicolumn{4}{|c|}{ Ergebnis verbessern } \\
\hline & & Einzelperson & Durchschnitt \\
\hline () & Konsum & $0,45 \mathrm{t}$ & $0,46 t$ \\
\hline () & Ernährung & $0,25 \mathrm{t}$ & $0,28 \mathrm{t}$ \\
\hline \multirow[t]{2}{*}{ (:) } & Flugverkehr & $0,00 \mathrm{t}$ & $0,14 \mathrm{t}$ \\
\hline & Öffentl. Verkehr & $0,00 \mathrm{t}$ & $0,01 \mathrm{t}$ \\
\hline (:) & Privatfahrzeuge & $0,07 \mathrm{t}$ & $0,29 \mathrm{t}$ \\
\hline  & Strom & $0,03 \mathrm{t}$ & $0,05 t$ \\
\hline (2) & Heizung & $0,91 \mathrm{t}$ & $0,37 \mathrm{t}$ \\
\hline & Öffentl. Emissionen & $0,13 \mathrm{t}$ & $0,13 \mathrm{t}$ \\
\hline \multicolumn{2}{|c|}{ Gesamt } & $1,83 \mathrm{t}$ & $1,72 \mathrm{t}$ \\
\hline \multicolumn{2}{|c|}{ Differenz } & \multicolumn{2}{|c|}{$0,11 \mathrm{t}$} \\
\hline
\end{tabular}

Ergebnis drucken

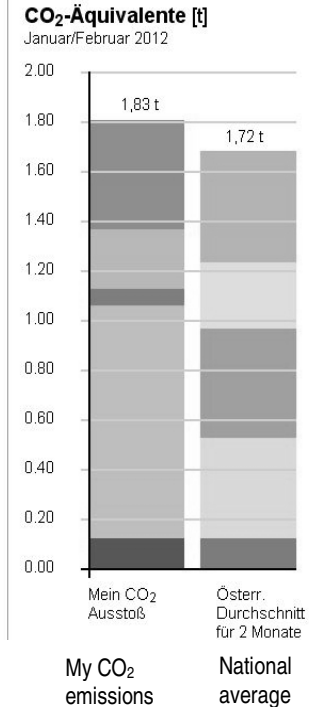

Fig. 1. Feedback on individual $\mathrm{CO}_{2}$ emissions 


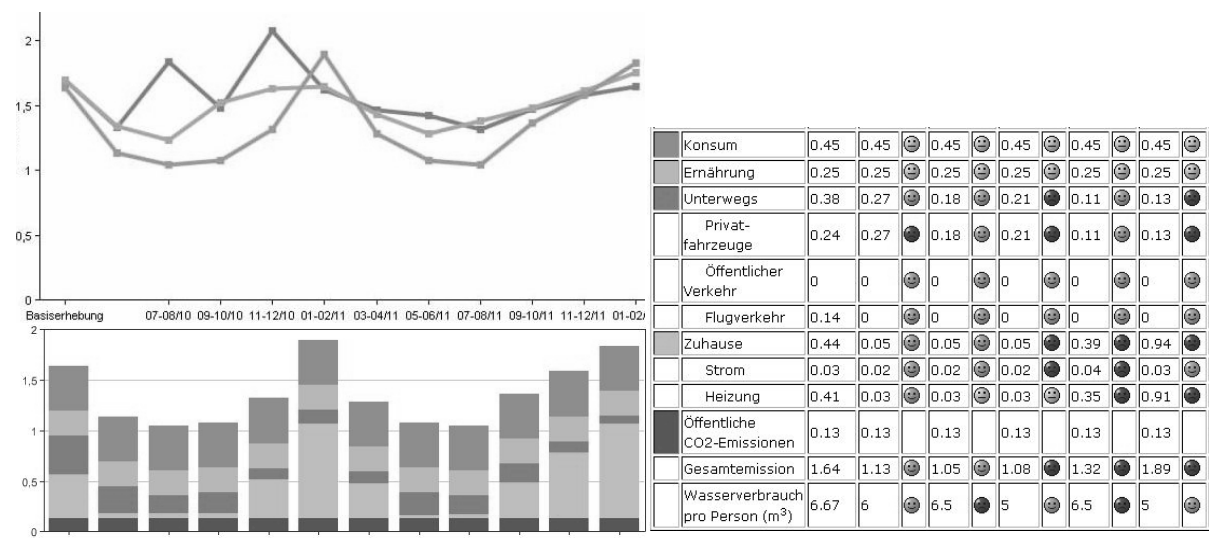

Fig. 2. Progression of individual $\mathrm{CO}_{2}$ balance in comparison to others

Note: The tool in Spain did not provide line-charts, emoticons and recall at any time

\section{$4 \quad$ Research Methods}

The empirical results in the next section stem from a survey among the citizen panels in six locations: Zaragoza, Pamplona, Bremen, Bremerhaven, Bregenz and Mariazell region. The surveys took place after eight rounds of the bimonthly measurements of consumption records (mainly in late autumn 2011). The majority was carried out online; "offliners" got postal questionnaires. A total of 286 valid replies were received which split into $70 \%$ onliners and $30 \%$ offliners. The distribution by country was as follows: Austria: 14\%; Germany: 29\%; Spain: 57\%.

An additional source was data on consolidated $\mathrm{CO}_{2}$ balances from the carbon calculator. This data was used for an overview on effects in terms of individual changes of $\mathrm{CO}_{2}$ balances over time and achievements of local climate targets. This analysis only included data from Austria and Germany to be able to consider participation processes of comparable long-term duration. By March 2012, 222 participants in Austria and Germany (i.e. $45 \%$ of a total of 496 initially registered) continuously participated and delivered data for the $\mathrm{CO}_{2}$ measurements; over nearly two years altogether.

\section{Empirical Results}

\subsection{Do Participants of Climate Initiatives Use the Eco-Feedback Offered?}

The first research question investigated the actual use of information feedback provided by the carbon calculator. Participants were asked "How often do you take a look at the results of your personal $\mathrm{CO}_{2}$ balance?" Table 1 shows the results which confirm the expectation that the participants of climate initiatives show interest in this sort of eco-feedback and used the information offered. 
Table 1. Frequency of viewing personal $\mathrm{CO}_{2}$ balance

\begin{tabular}{|c|c|c|c|c|c|c|}
\hline & & & Never & Seldom & $\begin{array}{l}\text { Frequently/ } \\
\text { after each entry }\end{array}$ & $\begin{array}{r}\text { Significance of } \\
\text { differences }\end{array}$ \\
\hline & & $\mathrm{N}$ & & & Percentages & \\
\hline Total & & 286 & 16 & 26 & 58 & \\
\hline \multirow[t]{3}{*}{ Country } & Austria & 43 & 9 & 21 & 70 & \multirow[t]{3}{*}{$* * *$} \\
\hline & Germany & 81 & 2 & 11 & 86 & \\
\hline & Spain & 162 & 25 & 35 & 41 & \\
\hline \multirow[t]{2}{*}{ Medium } & Offline & 88 & 0 & 10 & 90 & \multirow[t]{2}{*}{$* * *$} \\
\hline & Online & 198 & 23 & 33 & 44 & \\
\hline \multirow[t]{2}{*}{ Gender } & Women & 132 & 14 & 29 & 57 & \multirow{4}{*}{$* * *$} \\
\hline & Men & 145 & 16 & 23 & 61 & \\
\hline \multirow[t]{2}{*}{ Age } & below 40 & 54 & 39 & 26 & 35 & \\
\hline & $40+$ & 231 & 11 & 26 & 63 & \\
\hline \multirow[t]{2}{*}{ Education } & low & 76 & 12 & 18 & 70 & \multirow[t]{2}{*}{$*$} \\
\hline & high & 198 & 17 & 27 & 56 & \\
\hline
\end{tabular}

Notes: Chi square test, significance levels: $* * *: \mathrm{p}<0.001 ; *: \mathrm{p}>0.05$

Overall a clear majority of panellists made use of the opportunity to inspect their $\mathrm{CO}_{2}$ balances, at least from time to time; $58 \%$ even frequently or after each data entry. However, a closer inspection reveals significant differences by country, medium and age. Participants in Spain, particularly the onliners among them, viewed their personal balances less frequently than users in Austria and Germany. A similar difference concerns the participation medium: offliners reported much more frequent inspection (90\%) than onliners (44\%). The explanation seems to be twofold, pointing to the importance of effort and information presented: Offliners got a pro-active feedback via mail from support staff whereas onliners had to initiate the generation of their $\mathrm{CO}_{2}$ balances themselves. The graphical presentation of feedback information offered by the Spanish calculator was more limited. Users were not able to view the graphic representation online at any time, but had to print or save it for the records; and it lacked historic feedback over the complete monitoring period. Another difference concerns the significantly higher rate of frequent viewing of $\mathrm{CO}_{2}$ balances among older participants $(63 \%)$ compared to the younger ones $(35 \%)$. This is clearly linked to the medium effect explained and largely owed to the fact that older panellists were mainly offliners. The same explanation holds for a higher usage rate among participants with lower education. By contrast, men and women did not differ in their use of carbon-related eco-feedback.

\subsection{How Do Participants Assess Effects of Eco-Feedback?}

The survey results also offer information on self-assessments by citizen panellists on hypothesised effects of regular eco-feedback. Having practised regular personal $\mathrm{CO}_{2}$ balancing for more than one year, panellists were well equipped to reflect and report on their views on effects discussed in the literature. The results offer a rather positive picture (Table 2). The vast majority of users confirmed learning effects (86\%), awareness raising (89\%) and valuable guidance on points for improvement (82\%). 
Table 2. Participants' assessments of effects of regular feedback on personal $\mathrm{CO}_{2}$ balance

\begin{tabular}{|c|c|c|c|}
\hline & & $\begin{array}{l}\text { "not at at all / to } \\
\text { a little extent" }\end{array}$ & $\begin{array}{l}\text { "to a great or very } \\
\text { great extent" }\end{array}$ \\
\hline & $\mathrm{N}$ & Percentages & \\
\hline $\begin{array}{l}\text { Learning about } \mathrm{CO}_{2} \text { impacts of own } \\
\text { behaviour }\end{array}$ & 281 & 14 & 86 \\
\hline $\begin{array}{l}\text { Showing importance of own } \\
\text { behaviour } \\
\text { Helpful guidance on improving own }\end{array}$ & 282 & 11 & 89 \\
\hline $\mathrm{CO}_{2}$ balance & 277 & 18 & 82 \\
\hline $\begin{array}{l}\text { Comparison with others strengthens } \\
\text { own efforts in climate protection }\end{array}$ & 274 & 44 & 56 \\
\hline $\begin{array}{l}\text { Comparison with others shows own } \\
\text { efforts can be lessened }\end{array}$ & 275 & 59 & 41 \\
\hline
\end{tabular}

The opportunity to compare one's balance with others (panellists in the same region or country) was somewhat less appreciated. A slight majority (56\%) confirmed the presence of an effort enhancing effect. However, there were also clear indications of the boomerang effect reported elsewhere [21]: $41 \%$ of the respondents largely admitted that the results of comparing their personal $\mathrm{CO}_{2}$ balances to others led to the effect of lowering their own efforts.

\subsection{How Far Does the Participatory Setting Support Individual Eco- Feedback?}

An important test of the assumption that individual eco-feedback would be more effective when embedded in a collective participatory process is looking at the results of the participants' views on some aspects of this. One related finding is that more than two thirds of the panellists reported feelings of a community experience. Having been asked about specific effects of being part of a collective initiative, panellists confirmed most of them (Table 3): three quarters of participants agreed that the collective process alleviated barriers encountered at an individual level and that it strengthened individual efforts to change climate-related habits. The negatively formulated statement on the effect on one's personal $\mathrm{CO}_{2}$ reduction conveyed no clear result and will thus be compared to carbon calculator results later.

Table 3. Participants' assessments of effects of engagement in collective effort

\begin{tabular}{lrrr}
\hline & & $\begin{array}{l}\text { "not at all / } \\
\text { to a little } \\
\text { extent" }\end{array}$ & $\begin{array}{c}\text { "to a great / } \\
\text { very great } \\
\text { extent" }\end{array}$ \\
\hline & $\mathrm{N}$ & Percentages & \\
\hline Alleviates individual barriers & 268 & 24 & 76 \\
Has been irrelevant for own $\mathrm{CO}_{2}$ reduction & 269 & 46 & 54 \\
Strengthens change of individual habits & 268 & 25 & 75 \\
Believe in importance of further initiatives & 273 & 5 & 95 \\
\hline
\end{tabular}


Another consequence of participating in the collective climate initiative, on which the panellists agreed the strongest (95\%), was a shared belief in the importance of further common activities for climate protection. The interesting and significant relationship between community experience and the above reported effects additionally confirms for the beneficial role of the collective participation process: the presence of the effects tends to be higher, the stronger the community experience.

\subsection{What Are the Impacts Regarding Local Climate Targets?}

The initial aim of the citizen participation process was to trigger changing behaviours to achieve local climate targets. Our data on $\mathrm{CO}_{2}$-balances are a valuable source, in addition to assessments by participants, to examine whether this goal has been reached. Figure 3 reveals an ambivalent picture:

Around two-thirds of the participants show a reduced carbon footprint after nearly two years. Individually this group has achieved the local targets and the panel as a whole saved about 0.036 tons of $\mathrm{CO}_{2}$ per person within a two month period. This corresponds to the assumed positive effects of feedback. However, opposed to the improvements, there are also significant increases in $\mathrm{CO}_{2}$ emissions among the remaining third. While $\mathrm{CO}_{2}$ emissions decreased by $15 \%$ on average among the 146 cases with improved balances, they increased by $24 \%$ among the 76 cases with worsened balances. In effect, the smaller number with negative trends caused 0.15 tons more $\mathrm{CO}_{2}$ than the twice as big group with improved balances saved. Although the $\mathrm{CO}_{2}$ emissions were reduced in total, this triggered a significant rebound.

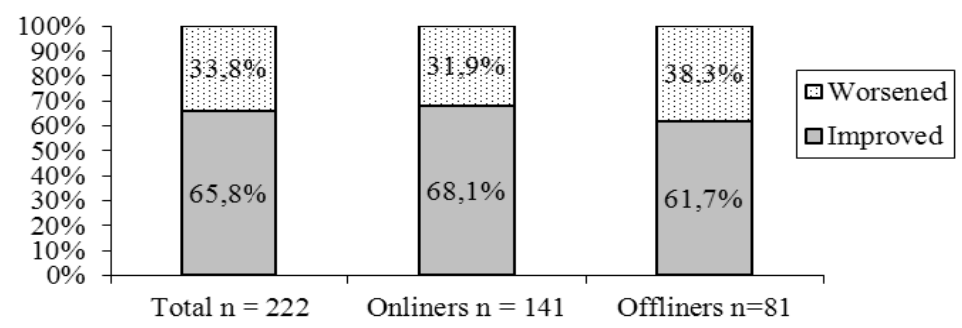

Fig. 3. Changes of individual $\mathrm{CO} 2$-balances over time

One reason is the lack of influence on individual energy consumption in one or more domains (e.g. no alterative transport options or no possibility to change heating system), which limits the capability to change behaviours and reduce energy usage. These results point to the complexity of behavioural patterns and social practices being shaped by a variety of factors.

\section{Summary and Conclusions}

As discussed in section 2, eco-feedback systems aim at inducing pro-environmental behaviour on individual levels based on two different rationales, i.e. rational choice or 
norm activation models. Pointing to limitations of individual choice, theories of social practices and system transition to sustainability cast such a focus into doubt. Ecological economists suggest participatory approaches allowing for social learning, deliberation and joint action to further sustainable development. Our empirical results from six European cities confirm synergies of eco-feedback and (e-)participation approaches built on information sharing, $\mathrm{CO}_{2}$ balancing and various forms of exchange offered by local governments collaborating with citizen panels on climate targets. In combination these three strands of (e-)participation establish collective social action which provides an important backing to individual efforts. The findings are encouraging but revealed certain limits as well:

Participants make use of eco-feedback via a carbon calculator if it is informative in a number of respects and available at any time. A strong majority of users reports effects from regular $\mathrm{CO}_{2}$ balancing exercises, such as learning about impacts, awareness raising and guidance for reducing emissions. The strategy of comparing one's own energy balance to others is less sweeping since it brings many users to abating their endeavours. Results though confirm the crucial role of community experience to enhance individual efforts and empowerment. This substantiates assumed potentials of participation approaches in contrast to individually practised eco-feedback. However, this evidence is based on participants' own assessments. Regular measurements of behaviours are additionally needed to be able to seriously assess impacts on climate targets. These data show that assumed positive effects of feedback are strongly dependent on individual conditions of living. Changing behaviours towards more sustainable life-styles is not a linear process because energy consumption levels oscillate due to the interplay of various factors that complicate individual attempts to alter routines (ranging from seasonal peculiarities to limited controllability of energy supply, transport services, household equipment, etc.). This supports the criticism of rational-choice focused eco-feedback approaches as they neglect important factors and social practices. Currently most of these systems have a clear focus on triggering behavioural changes on an individual level. However, altering social practices not merely rests upon adapting individual behaviour but is closely entangled with value systems and structural constraints. Sustainability as a normative concept implicates joint action. Hence, the combination of eco-feedback and (e-)participation including modes of collaborative action reveals a strong and yet unexploited potential to strengthen sustainable citizenship.

Acknowledgments. The paper is based on the collaboration with the Institute for Information Management Bremen (ifib) and the University of Zaragoza in the project e2democracy, funded by the Austrian Science Fund (FWF): I 169-G16 within the EUROCORES ECRP IV programme of the European Science Foundation (ESF).

\section{References}

1. Hazen, S.: Environmental democracy. Our Planet 8(6) (1997)

2. Edwards, P., Hindmarsh, R., Mercer, H., Bond, M., Rowland, A.: A Three-Stage Evaluation of a Deliberative Event on Climate Change and Transforming Energy. Journal of Public Deliberation 4(1), Article 6 (2008) 
3. Carson, L.: Growing up politically: conducting a national conversation on climate change. Australian Policy Online (2010), http: / / apo . org . au / commentary / growingpolitically-conducting-national-conversation-climate-change

4. Bechtold, U., Ornetzeder, M., Sotoudeh, M.: WWViews in Austria - reflecting a citizen consultation project from a Technology Assessment perspective. In: Rask, M., Worthington, R., Lammi, M. (eds.) Citizen Participation in Global Environmental Governance, pp. 107-122. Earthscan Publications, London (2012)

5. Spagnolli, A., Corradi, N., Gamberini, L., Hoggan, E.E., Jacucci, G., Katzeff, C., Broms, L., Jönsson, L.: Eco-Feedback on the Go. IEEE Computer 44(5), 38-45 (2011)

6. Bottrill, C.: Internet-based carbon tools for behaviour change. Environmental Change Institute. University of Oxford (2007)

7. Padgett, J.P., Steinemann, A.C., Clarke, J.C., Vandenbergh, M.P.: A comparison of carbon calculators. Environmental Impact Assessment Review 28, 106-115 (2008)

8. Arroyo, E., Bonanni, L., Selker, T.: Waterbot: exploring feedback and persuasive techniques at the sink. In: Proc. CHI 2005, pp. 631-639. ACM Press (2005)

9. Thieme, A., Lawson, S., Weeden, J., Olivier, P., Kraemer, N.: BinCam: Waste Logging for Behavioural Change. In: Proc. CHI 2011, Vancouver, BC, Canada (2011)

10. Froehlich, J., Dillahunt, T., Klasnja, P., Mankoff, J., Consolvo, S., Harrison, B., Landay, J.A.: UbiGreen: Investigating a Mobile Tool for Tracking and Supporting Green Transportation Habits. In: Proc. CHI 2009, pp. 1042-1052 (2009)

11. Darby, S.: The effectiveness of feedback on energy consumption. A review for DEFRA of the literature on metering, billing and direct displays. University of Oxford, Oxford (2006)

12. Froehlich, J., Findlater, L., Landay, J.: The Design of Eco-Feedback Technology. In: Proc. CHI 2010, Atlanta, Georgia, pp. 1999-2008 (2010)

13. Pierce, J., Schiano, D.J., Paulos, E.: Home, Habit, and Energy: Examining Domestic Interactions and Energy Consumption. In: Proc. CHI 2010, pp. 1985-1994 (2010)

14. Patel, S.N., Gupta, S., Reynolds, M.S.: The design and evaluation of an end-user deployable, whole house, contactless power consumption sensor. In: Proc. CHI 2010, pp. 2471-2480. ACM Press, New York (2010)

15. Betz, M., Schwartz, T., Ramirez, L.: Energy Awareness and Conservation through Pervasive Applications. In: Workshop at Pervasive 2010, Helsinki, Finland (2010)

16. Blake, J.: Overcoming the 'value-action gap' in environmental policy: tensions between national policy and local experience. Local Environment 4, 257-278 (1999)

17. Pyrko, J., Darby, S.: Conditions of behavioural changes towards efficient energy use - a comparative study between Sweden and the United Kingdom. In: ECEEE Summer Study 'Act! Innovate! Deliver! Reducing Energy Demand Sustainability, pp. 1791-1800 (2009)

18. Gupta, R., Darby, S.: Action research approach for gaining, and providing, feedback on domestic energy use to understand occupant behaviour, perceptions and expectations. In: Proc. Energy and People: Futures, Complexity and Challenges, Oxford (2011)

19. Lanzarone, G.A., Zanzi, A.: Monitoring gas and water consumption through ICTs for improved user awareness. Information, Communication \& Society 13(1), 121-135 (2010)

20. Schleich, J., Klobasa, M., Brunner, M., Gölz, S., Götz, K., Sunderer, G.: Smart metering in Germany and Austria - results of providing feedback information in a field trial. Working Paper Sustainability and Innovation, no. S 6/2011. Fraunhofer ISI, Karlsruhe (2011)

21. Thaler, R.H., Sunstein, C.R.: Nudge - Improving Decisions about Health, Wealth, and Happiness. Yale University Press, New Haven (2008)

22. Shove, E.: Beyond the ABC: climate change policy and theories of social change. Environment and Planning A 42(6), 1273-1285 (2010)

23. Garmendia, E., Stagl, S.: Public participation for sustainability and social learning: Concepts and lessons. Ecological Economics 69, 1712-1722 (2010) 